\title{
Fatigue Analysis of Large-scale Wind turbine
}

\author{
Yongli Zhu ${ }^{1, a}$ and David Lawson ${ }^{2}$ \\ ${ }^{1}$ Chongqing Vocational Institute of Engineering, Chongqing Engineering Polytechnic College, Chongqing, China; ${ }^{\mathrm{a} E-m a i l: ~}$ \\ 1348125719@qq.com
}

${ }^{2}$ Northern Melbourne Institute of TAFE

\begin{abstract}
The paper does research on top flange fatigue damage of large-scale wind turbine generator. It establishes finite element model of top flange connection system with finite element analysis software MSC. Marc/Mentat, analyzes its fatigue strain, implements load simulation of flange fatigue working condition with Bladed software, acquires flange fatigue load spectrum with rain-flow counting method, finally, it realizes fatigue analysis of top flange with fatigue analysis software MSC. Fatigue and Palmgren-Miner linear cumulative damage theory. The analysis result indicates that its result provides new thinking for flange fatigue analysis of large-scale wind turbine generator, and possesses some practical engineering value.
\end{abstract}

Keywords. Large-scale wind turbine generator; top flange; fatigue load spectrum; fatigue analysis

\section{Introduction}

Top flange of wind turbine generator is a key component connecting tower and main frame, it bears various alternating load from the top of wind turbine generator including wind load, dead-weight of generator etc., and fatigue damage easily occurs, flange fatigue damage directly influences on performance of entire WTGS, therefore, fatigue analysis of top flange plays a significant role of WTGS structure design.

According to design requirements of homemade MW-level WTGS, top flange as one key component of WTGS can't present fatigue failure within 20 years life cycle. Currently, domestic and foreign wind power manufacturing enterprises firstly establish simulation model of components with common fatigue analysis method during design process of WTGS, then ensure fatigue life of component in combination of engineering experience, this requires design staffs not only to utilize simulation software effectively, but also possess rich and practical engineering experience, the advantages of certification authority embodies herein as well [1].

German Lloyd's Register of Shipping (Germanisher Lloyd, abbreviation as GL)is one of top world classification societies, which offers a series of certification service like inspection of materials, components, complete appliance and wind power farm [2].GL Wind Power Norm is one of international universal WTGS design, manufacturing and certification norm, which was formulated by Lloyd's Register of Shipping with its years of design and manufacturing experience in wind turbine generator field, it possesses highly reference value, and it is one of main reference standard for domestic wind power certification institutes, at present, most wind power farms require the products passing GL certification when purchasing WTGS [3].

The paper takes top flange of some MW-level wind power generator as example, and firstly establishes finite element model of top flange connection system with finite element analysis software MSC.Marc/Mentat, analyzes fatigue strain of top flange, corrects S-N curve line 1 of flange materials based on GL norm, realizes simulation of flange fatigue working condition with WTGS design software Bladed, and obtains flange fatigue load spectrum in 
connection with rain-flow counting method, at last, it analyzes flange fatigue damage with fatigue analysis software MSC.Fatigue and Palmgren-Miner linear cumulative damage law.

\section{Analysis of finite element fatigue strain of top flange}

\subsection{Geometric model of top flange connection system}

It establishes overall modelling for top flange connection structure with Pro/E software, makes simplified treatment for the parts playing unimportant role of top flange, such as main framework base and some accessory equipment etc., the simplified geometrical model shown as Figure 1:

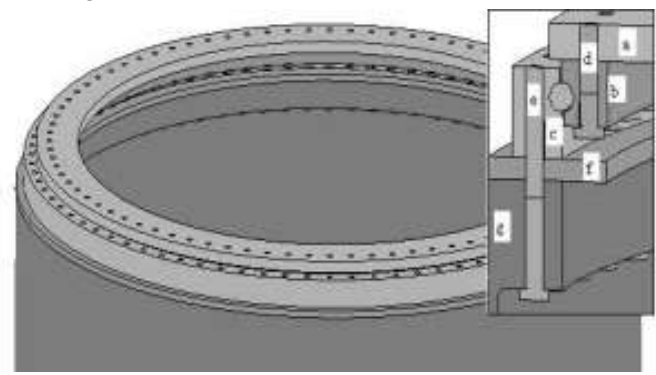

a- main frame, b-yaw bearing inner race, c-yaw bearing outer race, d-inner race bolt, e- outer race bolt, f-brake disc, g- top flange

Fig.1. Geometrical model of top flange system

\subsection{Flange finite element model}

The paper builds up top flange connection system in touch with finite element model by MSC.Marc/Mentat, model division adopts 8 nodes, hexahedron and No.7 unit, total units are 35,648, and total nodes are 389,788. Models include deformable contact bodies like main frame, yaw bearing, outer race screw, inner race screw, brake disc and top flange etc., materials of various component are made of low-alloy high-tensile structural steel, elasticity modulus quantity is $2.06 \times 10^{5} \mathrm{MPa}$, Poisson's ratio is 0.3 , density is $7.85 \times 10^{-9} \mathrm{t} / \mathrm{mm}^{3}$. Among these, flange material is forged part S355NL-Z25-0.

\subsection{Computation of flange fatigue strain}

When computing fatigue strain, it will respectively exert 6 limit loads(Fx, Fy, Fz, Mx, My, Mz), load setting shown as Table1:

Table 1. Limit load setting

\begin{tabular}{|c|c|c|c|c|c|c|}
\hline $\begin{array}{c}\text { Working } \\
\text { condition }\end{array}$ & $\mathrm{Mx}(\mathrm{Nmm})$ & $\mathrm{My}(\mathrm{Nmm})$ & $\mathrm{Mz}(\mathrm{Nmm})$ & $\mathrm{Fx}(\mathrm{N})$ & $\mathrm{Fy}(\mathrm{N})$ & $\mathrm{Fz}(\mathrm{N})$ \\
\hline Lc_Mx & $5 \mathrm{e} 9$ & 0 & 0 & 0 & 0 & 0 \\
\hline Lc_My & 0 & $-8 \mathrm{e} 9$ & 0 & 0 & 0 & 0 \\
\hline Lc_Mz & 0 & 0 & $-7 \mathrm{e} 9$ & 0 & 0 & 0 \\
\hline Lc_Fx & 0 & 0 & 0 & $-9 \mathrm{e}$ & 0 & 0 \\
\hline Lc_Fy & 0 & 0 & 0 & 0 & $7 \mathrm{e} 5$ & 0 \\
\hline Lc_Fz & 0 & 0 & 0 & 0 & 0 & $-3 \mathrm{e} 6$ \\
\hline
\end{tabular}


Strain distribution status of each working condition will be obtained from the analysis and computation of finite element analysis software MSC.Marc/Mentat, Limit fatigue strain of 6 limit loads are respectively 22.7Mpa, 16.58Mpa, 43.23Mpa, 90.04Mpa, 143.1Mpa, 58.73Mpa.

\section{S-N curve line and its correction}

S-N curve line indicates the relation between applied strain level and the strain experienced before fatigue failure of materials or strain circulation frequency [5]. S-N curve line is achieved from processing materials into standard component and making fatigue experiment. Although this method is reliable, a large amount of manpower and material resources will be consumed during design period, and test cycle is longer. GL norm regulates that it can utilize material parameters to imitate approximate S-N curve line replacing the obtained curve line [2] by experiment if fatigue experiment can't be made.

With log-log coordinate, S-N curve line is an ideal straight line [5].

Expression of S-N curve line in logarithmic coordinate system is:

$$
\log S=\frac{1}{m} \log K-\frac{1}{m} \log N
$$

(1)In Expression (1), $\mathrm{m}$ and $\mathrm{K}$ are parameters related to materials, loading method and strain ratio etc. [6]

Generally, S-N curve line in fatigue analysis gives survival probability of component as $P_{U}=50 \%$, on the basis of

GL Norm, it can make components possess survival probability as $P_{U}>97.7 \%$ and guarantee non-fatigue failure within 20 years life time as the design when making fatigue analysis of components of wind turbine generator. Therefore, when S-N curve line requires the correction, reference strain amplitude within finite lifetime shall multiply reduction coefficient $S_{P U}$, consequently, there is:

$$
\Delta \sigma_{A}^{*}=\Delta \sigma_{A} \cdot S_{P U}
$$

(2) When survival probability is $P_{U}>97.7 \%, S_{P U}=2 / 3$.

Considering some defects like chilling and cracks etc. in the flange forging process, it shall multiply forging defect coefficient $S_{t}$ :

$$
S_{t}=(t / 25)^{-0.1}
$$

(3) In Expression (3)[7], $t$ is thickness impact coefficient.

Finally, reference strain amplitude of corrected S-N curve line within finite lifetime area is:

$$
\Delta \sigma_{A}^{*}=2 \cdot \Delta \sigma_{A} \cdot \frac{S_{P U} \cdot S_{t}}{\gamma_{m}}
$$

(4)In Expression (4) [7], $\gamma_{m}$ is partial security coefficient of material, $\gamma_{m}$ takes 1.265.

When flange strain circulation frequency is more than limit strain circulation frequency, S-N curve line shall prolong with slope parameter $\frac{1}{1-2 m}$.

In fatigue analysis software MSC. Fatigue, S-N curve line of flange material is corrected with PTMAT module.

\section{Fatigue load spectrum}




\subsection{Fatigue load simulation}

For fatigue life analysis, it shall firstly know load situation of component. The main source creating WTGS fatigue load is alternating load produced during the wind wheel running which is caused by mass eccentricity of wind wheel as well as alternating load caused by blade gravity when wind wheel turns.

Concerning analog simulation of WTGS fatigue load, it must firstly comprehend fatigue working condition of WTGS. According to GL Norm, WTGS has 6 large fatigue load working condition, including 46 fatigue load sub-working condition. For each fatigue working condition, it will compute fatigue load at the hub center, then make equivalent treatment to obtain fatigue load time sequence curve line of top flange.

\subsection{Fatigue load spectrum}

Fatigue load time sequence curve line records the process of fatigue load varying with time, which is a series of irregular data, and fatigue load spectrum [8] is acquired with certain amplitude statistical counting method for probability statistical disposition. Load spectrum presents like graph, digital form or matrix of occurrence frequency with size of random load. Based on regulation of GL Norm, fatigue analysis computation can make simplified fatigue analysis with equivalent constant amplitude fatigue load spectrum.

The paper adopts rain-flow counting method to treat data. At present, rain-flow counting method is the most popular calculation method in fatigue design and experiment, and it can comprehensively reflect entire process of immediate load, possess stricter mechanics base[9,10], and easily realize the programming in the computer.

In Bladed software, it will adopt rain-flow counting method to compute flange random load, and obtain a series of WTGS fatigue load as well as load circulation frequency, and acquire total circulation frequency of load within WTGS life cycle according to wind speed characteristics and required life time of WTGS design, then it can draw time-domain equivalent fatigue spectrum of wind turbine. There are 46 fatigue load sub-working conditions for top flange, and each sub-working condition includes 6 equivalent fatigue load spectrums, for example, equivalent load spectrum of sub-working condition DLC1.2ac shown as Figure 2. Regularization of equivalent fatigue load spectrum can achieve equivalent fatigue load spectrum.

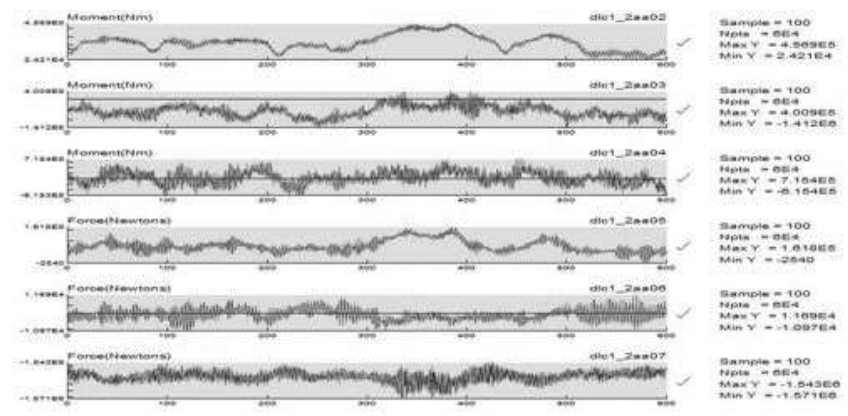

Fig.2. Working condition DLC1.2 ac time-domain equivalent load spectrum

\section{Flange fatigue analysis}

Flange finite element analysis model and flange fatigue strain result are imported into fatigue analysis software MSC. Fatigue, limit fatigue strain computed with limit load is treated as unitization, equivalent weight fatigue load spectrum under each working condition is connected with correspondent fatigue strain analysis result. Frequency is different under each working condition each year, therefore, it needs set yearly circulation indexes of each fatigue working condition. Moreover, S-N curve line shall be set, the paper utilizes the corrected S-N curve line in second section. Equivalent fatigue load spectrum setting is uploaded with sinusoid mode. Fatigue analysis results shown: it can see that maximum fatigue damage value of top flange is 0.0356 within one year, maximum value presents at the round corner 
connecting flange and yaw bearing, fatigue damage in other positions is very small, and it can be neglected to count. According to Palmgren-Miner cumulative damage theory, cumulative damage of wind power turbine within its life cycle can't surpass 1 , or the component will lead to fatigue failure [11].Fatigue damage value of top flange is calculated as:

$$
D_{20}=20 \times 0.0356=0.712<1
$$

Therefore, fatigue damage will not take place on top flange within life cycle, and it will meet the requirements for 20 years of life cycle.

\section{Conclusion}

The paper takes top flange of some large-scale wind power turbine generator as an example, and discusses top flange fatigue damage analysis method of some wind power generator, the method constructs finite element model of top flange connection system with finite element analysis software MSC.Marc/Mentat, analyzes top flange fatigue strain, realizes load simulation of flange fatigue working condition with Bladed software, acquires flange fatigue load spectrum, finally achieves top flange fatigue analysis with fatigue analysis software MSC.Fatigu and Palmgren-Miner linear cumulative damage theory. The analysis method offers a new thinking way for flange fatigue analysis of large-scale wind power generator, and it possesses some practical engineering value.

\section{References}

1. H. Liu, WTGS System Dynamics Model and Key Component Optimization Study, Chongqing University, (2009)

2. Germanischer Lloyd Wind Energie GmbH. Guideline for the Certification of Wind Turbines.German: Germanischer Lloyd Wind Energie GmbH, (2010)

3. C. Y. Zhou, H. X. Zheng, H. Q. Luo, etc. Application and Samples of MSC.Fatigue Analysis, Beijing: Science Press, (2005)

4. G. J. Wang, Guidance of MSC. Fatigue Analysis Example, Beijing: China Machine Press, (2009)

5. B.N. Shi, MW-level WTGS Main Shaft Lifetime Analysis. Power Grid and Clean Energy 8(2010)

6. Y. Li, Ship Hull Fatigue Analysis Based on JBP Norm. Dalian University of Technology, (2006)

7. S.M. Li, Mechnical Fatigue and Reliability Design, Beijing: Science Press.

8. H.Q. Sheng, Research on Converter Fatigue Load Spectrum. Acta Mechanica Solida Sinica, 29(2008)

9. T.L. Li, S.J. Zou, D. Sun, etc. New Method of Airplane Fatigue Load Spectrum Analysis, Mechanical Science and Technology, 6(2006)

10. A.K. Khosrovanch, N.E. Dowling, Fatigue Loading History Reconstruction Based on the Rain-Flow Technique, International Journal of Fatigue, (1990).

11. A.M. Cai, D.T. Zhang, H.T. Dai, Wind Turbine Fatigue Analysis. 2009 Annual Conference of Clean and Efficient Coal-fired Power Generation Technology Collaboration Network, 10(2009) 